\title{
Shadow Sort - A Hybrid Non-dominated Sorting Algorithm
}

\author{
Nicholas Tränkle and James Bekker
}

\begin{abstract}
Presented is a novel, hybrid, non-dominated sorting algorithm called Shadow Sort. The algorithm was developed bearing in mind real-world, practical requirements of nondominated sorting algorithms. The majority of non-dominated sorting algorithms are employed in conjunction with a multiobjective optimization algorithm, many of which make use of population-based meta-heuristic techniques. The outputs of each population-based meta-heuristic iteration typically include hundreds if not thousands of solutions which need to be sorted in order to prime the next evolutionary iteration. However, of all the solutions proposed by any given meta-heuristic iteration, only the solutions which make up the first, second and sometimes third Pareto set are of any use. Therefore, Shadow Sort approaches all non-dominated sorting by eliminating dominated solutions as early as possible, rather than processing all solutions in order to find their respective Pareto set. Shadow Sort was developed in Matlab, and is compared to Efficient Non-nominated Sort, Best Order Sort and Merge Non-dominated Sort, all of which it outperformed. Several cases of Shadow Sort are proposed based on the number of objectives in the population.
\end{abstract}

Index Terms-Evolutionary algorithms, multi-objective optimization, non-dominated sorting.

\section{INTRODUCTION}

$\mathbf{I}$ $\mathrm{N}$ multi-objective optimization with population-based meta-heuristics, non-dominated sorting algorithms are needed to rank solutions of populations to find the nearoptimal solutions to serve as input for subsequent iterations. If these algorithms are inefficient, they could be detrimental to the overall performance of an optimization meta-heuristic. Non-dominated sorting algorithms are often used in conjunction with meta-heuristics and they form part of an active field of research, with some of the latest algorithms being proposed as recently as the year 2020. Most non-dominated sorting algorithms attempt to improve computational times by minimizing the number of necessary comparisons. However, the majority of existing algorithms still require that all objectives are used during solution comparisons in order to ensure that dominance exists. Even the most recent non-dominated sorting algorithms apply methodologies which result in all solutions being assigned to their respective solution sets. Whilst comprehensive, this naturally requires more computations and time. In this study, we present an algorithm called Shadow Sort, which has been developed with the primary goal of reducing these costly comparisons. We assume the reader is acquainted with metaheuristics, multi-objective optimization (MOO) and ranking methods of meta-heuristics when doing MOO.

Nicholas Tränkle and James Bekker are both with the Department of Industrial Engineering, Stellenbosch University, South Africa, 7600. e-mail: jb2@sun.ac.za.

\section{Previous Work}

Much research has gone into the design and optimization of non-dominated sorting algorithms. Three of the latest and best performing algorithms are Efficient Non-dominated Sort (ENS-SS) [1], Best Order Sort (BOS) [2] and Merge Nondominated Sort (MNDS) [3]. These three algorithms were published in 2015, 2016 and 2020 respectively, and in their publications, are compared to various other non-dominated sorting algorithms where they were proven to be superior. Subsequently, Shadow Sort is compared to these three algorithms, and via transitive dependency, is not explicitly tested against lower performing algorithms.

\section{A. Efficent Non-dominated Sort}

Zhang, Tian, Cheng and Jin [1] took a closer look at the results of a dominance comparison taking place. They concluded that, given two solutions $p_{m}$ and $p_{n}$, a dominance comparison would result in one of four possible cases:

- Case 1: one of the points dominates the other, i.e., $p_{m} \prec p_{n}$ or $p_{n} \prec p_{m}$.

- Case 2: $p_{m}$ and $p_{n}$ are non-dominated, and both belong to the same and current Pareto set $F_{i}$.

- Case 3: $p_{m}$ and $p_{n}$ are non-dominated, and both belong to the same Pareto set $F_{i}$, but where $F_{i}$ is not the current set.

- Case 4: $p_{m}$ and $p_{n}$ are non-dominated, but belong to different Pareto sets.

The developers of ENS further explained that Case 1 comparisons contained some necessary comparisons (comparisons between solutions from different sets), all Case 2 comparisons are necessary, but that no Case 3 and Case 4 comparisons are necessary. Accordingly, the authors report that the sum of the necessary comparisons in Case 1 and Case 2 is the theoretical minimum number of required dominance comparisons for any non-dominated sorting algorithm. The ENS approach differs from most non-dominated sorting methods by determining the set to which each solution belongs, whereas other approaches generally cycle through the entire (unassigned) population per set desired. ENS has space complexity $\mathcal{O}(1)$. Time complexity depends on the approach used for the specific ENS implementation: for $M$ objectives and a population of size $N$, best case time complexity for ENS (SS) is $\mathcal{O}(M N \sqrt{N})$ and worst case time complexity is $\mathcal{O}\left(M N^{2}\right)$.

\section{B. Best Order Sort}

Roy, Islam and Deb [2] proposed Best Order Sort (BOS) which is designed to reduce the number of dominance comparisons in the worst case. The idea is to reduce the number 
of comparisons between a new incoming solution requiring evaluation against already assigned solutions. Since a given solution may be ranked in different positions over the various objectives, the algorithm tries to use the objective with the shortest list of solutions which are not worse than the incoming solution. Mishra, Saha and Mondal [4] analysed BOS and found that the algorithm did not perform well when duplicate solutions were present in the population. BOS has space complexity $\mathcal{O}(M N)$ while best case time complexity for $\mathrm{BOS}$ is $\mathcal{O}(M N \log N)$ and worst case time complexity is $\mathcal{O}\left(M N \log N+M N^{2}\right)$

\section{Merge Non-dominated Sort}

Moreno, Rodriguez and Nebro [3] proposed Merge Nondominated Sort (MNDS). MNDS sorts the solutions along all the objectives, each time creating an objective dominance set (ods) for each solution. Each solution's ods is comprised of all other solutions which dominate the current solution along that specific objective. The algorithm then calculates each solution's dominance set $(s d s)$ by finding the intersection of the current $o d s$ and the solution's $s d s$. This dominance set comprises all other solutions in the population which dominate the current solution. Best case time complexity for MNDS is $\mathcal{O}(N \log N)$ and worst case time complexity is $\mathcal{O}\left(M N^{2}\right)$

\section{SHADOW SORT}

The primary idea behind Shadow Sort (SS) is to eliminate unnecessary solution comparisons without needing to explicitly compare all objective values. This idea is also used in MNDS where each solution keeps a list of other solutions which dominate it. Conversely, however, Shadow Sort processes solutions in a strategic order, each time identifying all solutions which it dominates. By doing so, the dominated solutions will be identified as such once they are due to be processed and are skipped as they are of no use to the researcher. The name Shadow Sort is analogous to the algorithm's method of operation, namely by excluding solutions in the population which fall into the current solution's "shadow". Shadow Sort is considered a hybrid algorithm, as it capitalises on the population's properties to sort it efficiently. Therefore, there are four different sorting methods, depending on the number of objectives of the problem; Case I for two objectives, Case II for three objectives, Case III for four to 17 objectives, and Case IV for more than 17 objectives. A key property of Shadow Sort is that it is designed with the operational requirements of many multi-objective optimization algorithms in mind, namely to provide the algorithm with the best performing (rank 1) non-dominated set. In order to supply this information in the fastest way possible, Shadow Sort focuses exclusively on one non-dominated rank set at a time.

Next, each case will be presented along with its pseudocode, as well as an explanation of why each case is appropriate. For ease of explanation, all populations are assumed to have $N$ solutions and minimization of all objectives is assumed without loss of generality. For each case, the population is first sorted along the first objective before a working copy of the original population is made. Since Shadow Sort makes use of solution positioning relative to one another, sorting along the first objective enables each solution to be given a reference number $p$ which is then used to identify it going forward. The four cases are subsequently presented.

\section{A. Case I}

Case I is used for populations with two objectives. The algorithm begins by sorting the population according to the first objective. Ties are broken by considering the second objective. Since the solution with the best first objective is necessarily a member of the Pareto set, it is marked as such, and its second objective value is set as a flagging value. The algorithm then proceeds to compare the next solution's second objective value against the flagging value. If the next solution's second objective value is equal to or worse than the flagging value, the solution is ignored and the next solution is considered. If the solution's second objective value is better than that of the flagging value, the solution is marked as a Pareto solution and its second objective value becomes the new flagging value. Once the algorithm has completed this process for all solutions, the entire first Pareto set will have been identified.

A minor adjustment can be made to the algorithm to find additional Pareto sets, that is, solutions sets of which the values are dominated by one solution of the first set, in which no solutions are dominated. Instead of saving only one flagging value, two or more flagging values may be considered. If a solution is found to be dominated by a preceding solution on a specific Pareto set, then it will be considered for subsequent sets as stipulated by the analyst. Best case time complexity for SS Case I is $\mathcal{O}(N \log N)$ and worst case time complexity is $\mathcal{O}\left(N^{2}\right)$. The pseudo-code for Case I is provided in Algorithm 1 .

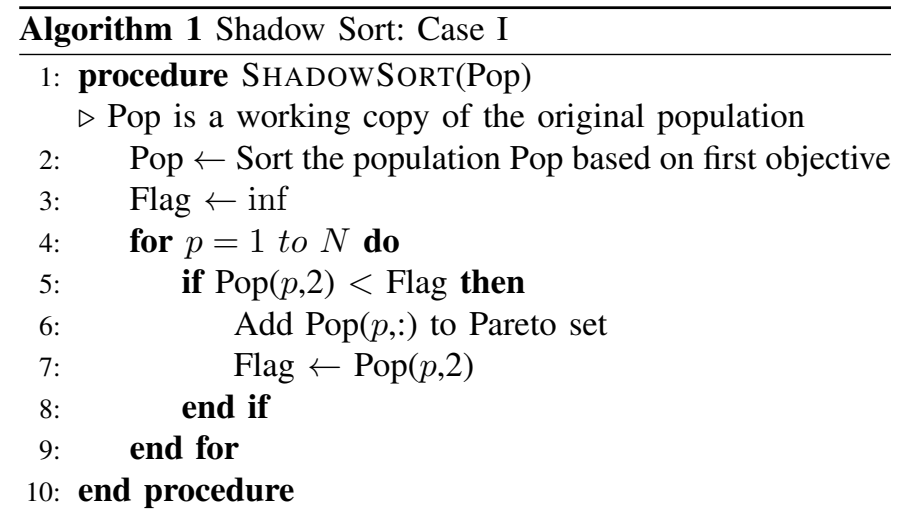

\section{B. Case II}

A population containing three objectives can be sorted by Shadow Sort with the first objective used to provide the order of processing for the remaining two objectives. The algorithm (pseudo-code provided in Algorithm 2) begins by sorting the population according to the third objective, and then replacing the population's actual values in column 3 with integers from 1 to $N$. Should there be solutions with identical values for objective 3 , then ties are broken by the other objectives as 
required. This process is then repeated for objectives 2 and 1 . Similarly to Case I, if there are duplicate solutions, one such solution is kept in the population and the rest are discarded. The result is an $N \times 3$ matrix with columns populated with integers 1 to $N$. The algorithm then creates a sparse matrix with ones using the contents of the columns of objectives 2 and 3 of solution $p$ as row and column indices respectively. The reason for using a sparse matrix is to reduce memory requirements, as only one $N^{t h}$ of the matrix will be populated. The algorithm then proceeds to process the solutions according to the ordered first objective. A solution whose position in the sparse matrix is read as 1 is added to the Pareto set. The sparse matrix is then updated by setting all points from solution $p$ 's second objective reference and below, and solution $p$ 's third objective and to the right to zero. This is analogous to eliminating all solutions which fall within the shadow of solution $p$. Once the algorithm has checked all $N$ solutions, the entire first Pareto set is found. Should additional sets be required, the process is repeated with the remaining solutions not added to the first Pareto set. Case II proves to be an especially efficient non-dominated ranking technique when the majority of the solutions in the population are non-dominated solutions. This is a likely scenario during the later stages of a multi-objective optimization operation when a diverse solution set evolves. Best case time complexity for SS Case II is $\mathcal{O}(N \log N)$ and worst case time complexity is $\mathcal{O}\left(N^{2}\right)$.

A small problem solved by Case II is shown in Fig. 1 with eight solutions labeled A-H. As per Algorithm 2, the population is sorted along each of the three objectives, each time replacing the actual values with integers 1 to $N$. The result of this can be seen in the table on the left of Fig. 1(i) which provides a reference for a three-dimensional problem with eight solutions. The algorithm then creates a two-dimensional sparse matrix by using the solution's objective 2 and 3 values as indices. Fig. 1 ( $i$ ) shows the eight solutions placed in the matrix. In this example, the solution identities $\mathrm{A}$ to $\mathrm{H}$ are shown for ease of explanation, whereas the real algorithm populates the sparse matrix with ones. With the sparse matrix initialised, the algorithm uses objective 1 to indicate the order in which the solutions should be processed. The solution with the smallest first objective value is solution A. Using solution A's objective 2 and 3 values as row and column indices, the algorithm checks the value at this position on the sparse matrix and will find a ' 1 '. This indicates that the solution is nondominated, and so it is added to the Pareto set. Next, as per line 12 of Algorithm 2, all sparse matrix entries to the right of and above $\mathrm{A}$ are then reassigned the value zero (or as indicated by Fig. 1(i), greyed out). In doing so, all solutions which are dominated by solution A are marked as such without needing to explicitly evaluate the dominance relationship. Solution B is the next to be evaluated and its value in the sparse matrix is checked as before. Since the value at B was set to zero during the processing of solution A, B is recognised as dominated and is not further considered. This process continues for the remaining solutions and is illustrated in Fig. 1 (iii) to (viii).

During performance testing, it was shown that, given a randomly generated population (as may be experienced during early stages of a multi-objective evolutionary algorithm execu-

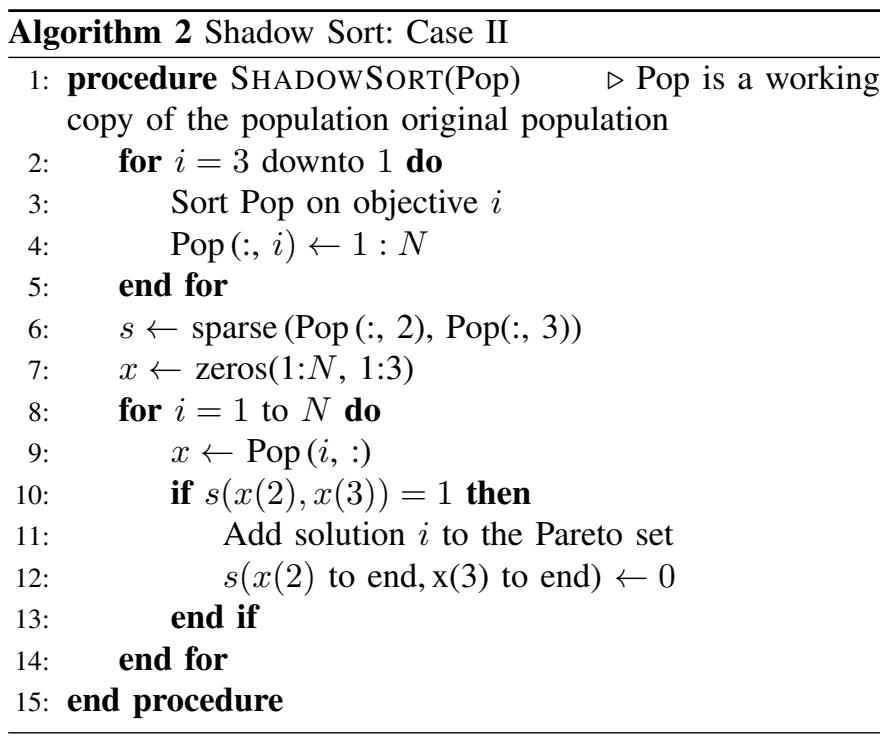

tion) there are cases where Shadow Sort would achieve faster performance if three-objective problems were sorted using Case III which is introduced in the next section. However, as the population matures and the majority of the solutions turn out to be rank 1 solutions, Case II turns out to be considerably more efficient for three-objective problems. In fact, the strategy employed in this case is considered by the authors to be an important area where future work should be focused, namely developing a means to create an efficient, multi-dimensional sparse array.

\section{Case III}

To explain this case, simple data structures are provided in Fig. 2. If four or more objectives are present, the creation, accessing and writing of a higher dimension sparse matrix as in Case II becomes too computationally expensive for the gain in memory saved. Case III therefore creates two $N \times M$ matrices which are used in conjunction for dominance checking. The first matrix Key is created in the same manner as in Case II. The result is a matrix whose columns are populated with integers 1 to $N$, with any cell indicating the rank of its respective far left solution in that particular objective. The second matrix called Standing is created by sorting the rows of Key along column $j \in[2, M]$, and then replacing the contents of column $j$ with the contents of column 1 of Key.

An example of this mapping is given in Fig. 2. For ease of explanation in the example illustration, the letters A to $\mathrm{J}$ are appended on the left of Key as a solution's identity. In the actual algorithm, this is not done as the integer in Key's column 1 is used as the solution's identity. Key can be interpreted as follows: if the solutions $k \in[1,10]$ of the original population had to be sorted along any objective $m \in[i, i v]$, then the rank of solution $k$ in objective $m$ can be found in cell $(k, m)$. The interpretation of Standing is as follows: if the solutions $k \in$ $[1,10]$ of the original population had to be sorted along any objective $m \in[i, i v]$, then the solution of rank $k$ in objective $m$ can be found in cell $(k, m)$. Thus, looking at solution 1 (A) in $K e y$, we see that its rank in objectives $i, i i, i i i$ and $i v$ are 1 , 


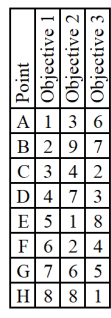

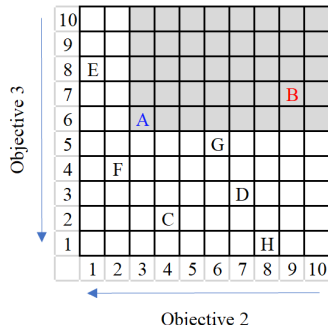

(i)

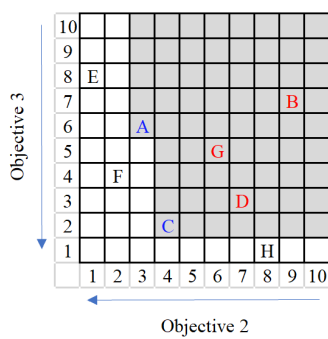

(iii)
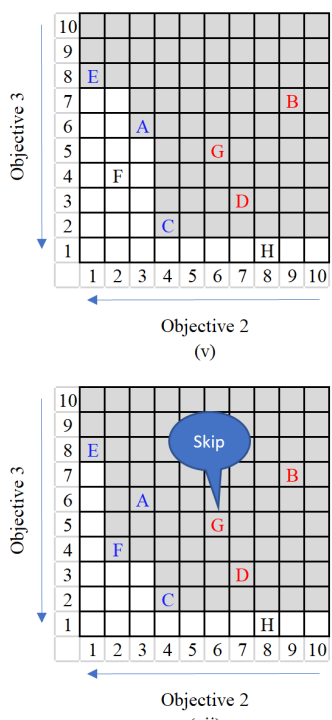

(vii)

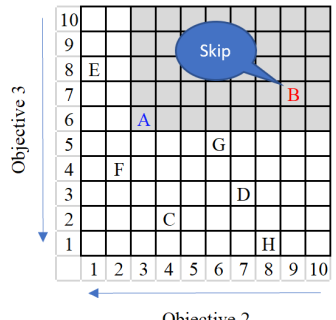

(ii)

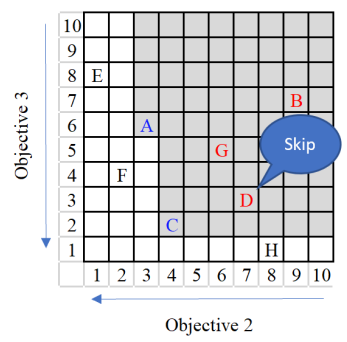

(iv)
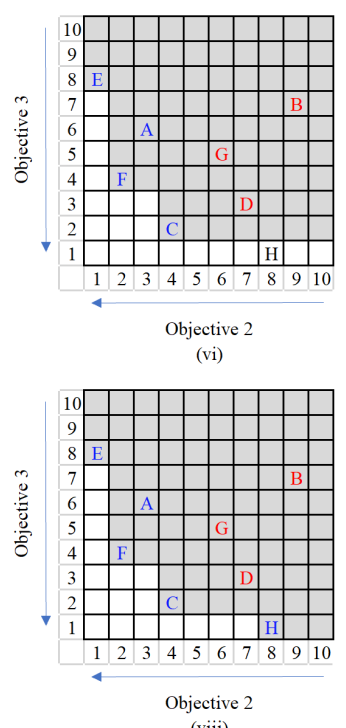

(viii)
Fig. 1. An illustrative example of Case II sorting non-dominated solutions in a three dimensional population.

2, 10 and 9 respectively. Looking at Standing, we see that if the population had to be sorted along objectives $i, i i, i i i$ and $i v$, the solutions with rank 1 are $A, J, J$ and $I$ respectively.

A column array called Flag is used as an indicator for whether or not a solution has been dominated by a preceding solution. During initialisation, all values of Flag are set to zero. Fig. 3 is provided to illustrate the workings of Case III: for each solution $k$ in column 1 of Key, its corresponding position in Flag is checked. If the solution's value in Flag is zero, the solution is marked as a non-dominated solution. Alternatively, if a solution's value in Flag is one or more, it is recognised as dominated and is skipped. Next, for $j \in[1, M]$, all solutions which are dominated by the current solution are identified. This is achieved by using Key and Standing in conjunction to manipulate the values of Bitmat. Bitmat is a binary array which is created to allow for efficient binary operations such as intersection or union comparisons. Since all values in Key

\begin{tabular}{|c|c|c|c|c|c|c|c|c|}
\hline & \multicolumn{4}{|c|}{ Key } & \multicolumn{4}{|c|}{ Standing } \\
\hline & $\overline{\mathrm{i}}$ & ii & iii & iv & $\mathrm{i}$ & ii & iii & iv \\
\hline $\mathrm{A}$ & 1 & 2 & 10 & 9 & $\bar{A}$ & $\mathbf{J}$ & $\mathrm{J}$ & I \\
\hline B & 2 & 4 & 8 & 5 & B & $\mathrm{A}$ & $\mathrm{D}$ & $\mathrm{F}$ \\
\hline $\mathrm{C}$ & 3 & 3 & 9 & 6 & $\mathrm{C}$ & $\mathrm{C}$ & $\mathrm{F}$ & $\mathrm{E}$ \\
\hline D & 4 & 9 & 2 & 8 & D & B & I & $\mathrm{J}$ \\
\hline$E$ & 5 & 8 & 6 & 3 & $E$ & $\mathrm{~F}$ & $\mathrm{G}$ & B \\
\hline $\mathrm{F}$ & 6 & 5 & 3 & 2 & $\mathrm{~F}$ & $\mathrm{H}$ & $\mathrm{E}$ & $\mathrm{C}$ \\
\hline $\mathrm{G}$ & 7 & 7 & 5 & 7 & $\mathrm{G}$ & $\mathrm{G}$ & $\mathrm{H}$ & $\mathrm{G}$ \\
\hline $\mathrm{H}$ & 8 & 6 & 7 & 10 & $\mathrm{H}$ & $E$ & B & $\mathrm{D}$ \\
\hline I & 9 & 10 & 4 & 1 & $\mathrm{I}$ & $\mathrm{D}$ & $\mathrm{C}$ & A \\
\hline$\overline{\mathrm{J}}$ & 10 & 1 & 1 & 4 & $\bar{J}$ & I & $\bar{A}$ & $\overline{\mathrm{H}}$ \\
\hline
\end{tabular}

Fig. 2. An example of matrices Key and Standing

and Standing have been replaced by integers, these values can be used to specify index positions of a matrix. First, the value of cell $(k, j)$ in Key is found, let us call it $x$. In Fig. 3a, $x=$ 6. This value is incremented by one (Algorithm 3, line 22), and in turn, is used to reference a set of points in Standing, namely cells $(x+1$ to end, $j)$. In Fig. 3 a, these are cells $\mathrm{G}$, $\mathrm{H}, \mathrm{I}$ and $\mathrm{J}$. These values will indicate specific indices within Bitmat which need to be set to True. In Fig. 3a, these are the cells in Bitmat which are set to 1. As explained above, this procedure is repeated for $j \in[1, M]$ (see Fig. $3 b$ to Fig. 3d.). Finally, each row of Bitmat is assessed as a logical conjunction operation, i.e. if the entire row consists of 1's (true's), then the result of that row is 1 (true). If at least one column contains a 0 (false), the result of that entire row is 0 (false). Flag is then set to the logical disjunction of Flag and the above logical conjunction operation of Bitmat before incrementing $k$ to process the next solution (see Fig. 3e). In Fig. 3e, solutions $\mathrm{G}$ and $\mathrm{H}$ have a value of 1 in Flag, and so they are recognised as dominated and will not be processed.

Similarly to Case I \& II, should multiple Pareto sets be required, then the procedure is simply repeated after removing the already found non-dominated solutions. Best case time complexity for SS Case III is $\mathcal{O}(N \log N)$ and worst case time complexity is $\mathcal{O}\left(N^{2}\right)$.

\section{Case IV}

The fourth case of Shadow Sort is used for populations that have 17 or more objectives. The reason for this is to take advantage of a statistical property of populations having this many objectives, namely that the probability of any one solution having a superior ranking on at least one objective increases as the number of objectives increases. The strategy of Case IV is therefore to find the worst performing objective for each solution $p$, and then perform a traditional dominance check between $p$ and all solutions worse than solution $p$ on that objective. In doing so, Shadow Sort minimizes the number of dominance checks required whilst still enabling the algorithm 


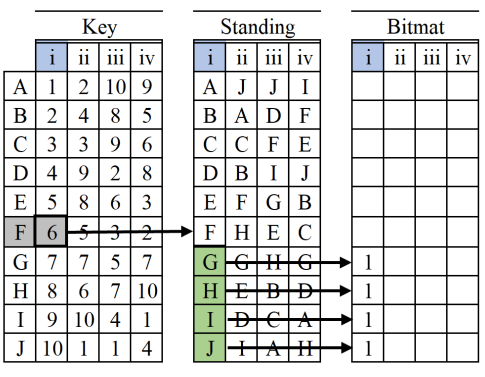

(a) Case IIIa

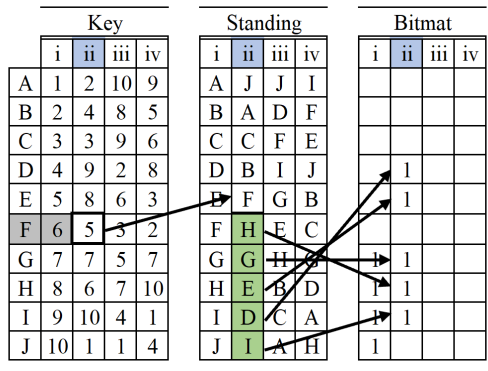

(b) Case IIIb

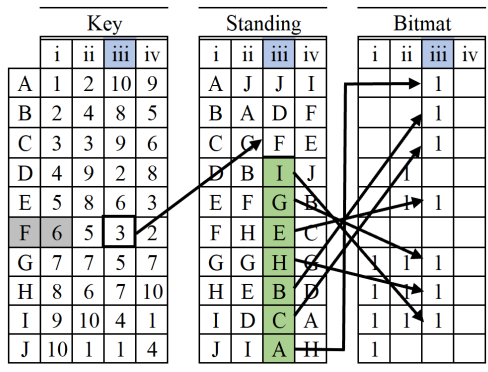

(c) Case IIIc

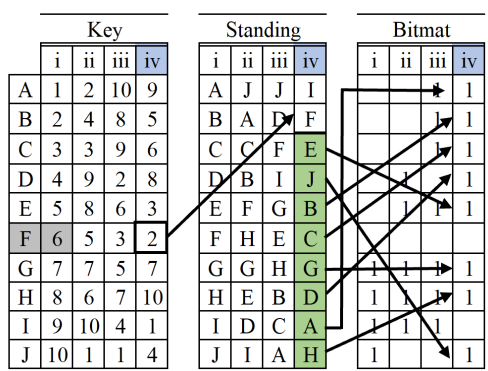

(d) Case IIId

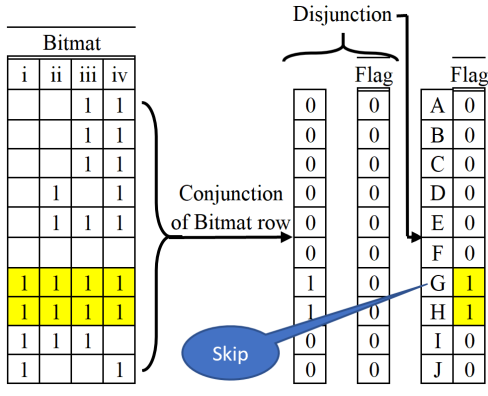

(e) Case IIIe

Fig. 3. Simple data structures to explain Case III

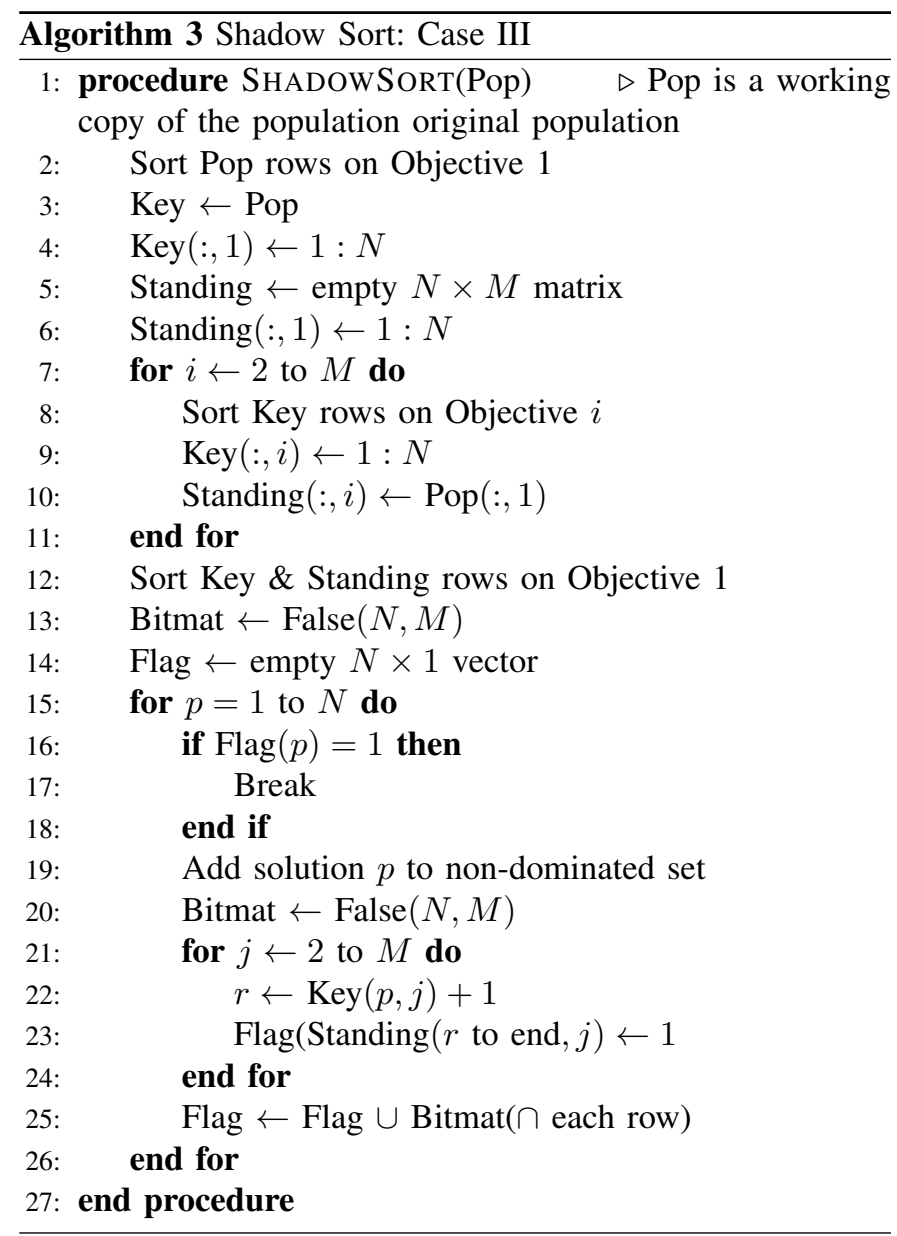

to identify all solutions which could be dominated by solution $p$. Thereafter, similar to Cases I to III, all dominated solutions are marked as such and are not processed by the algorithm later. Where dominance between two solutions needs to be assessed in Case IV, the same dominance check employed by [2] in their paper is used. This dominance check is presented in Algorithm 4. The pseudo-code for Case IV is presented in Algorithm 5. Best case time complexity for SS Case IV is $\mathcal{O}(N \log N)$ and worst case time complexity is $\mathcal{O}\left(N^{2}\right)$.

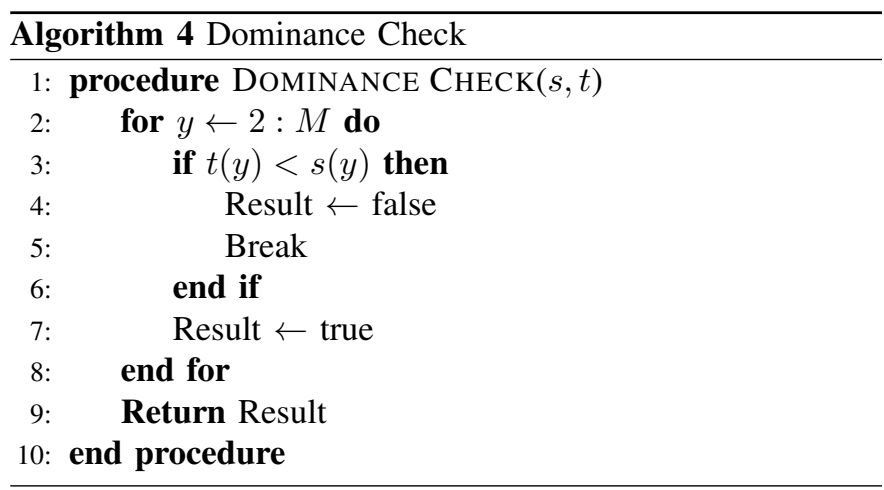

\section{EXPERIMENTAL RESUltS}

The proposed algorithm is compared against three existing non-dominated sorting algorithms - Efficient Non-dominated 


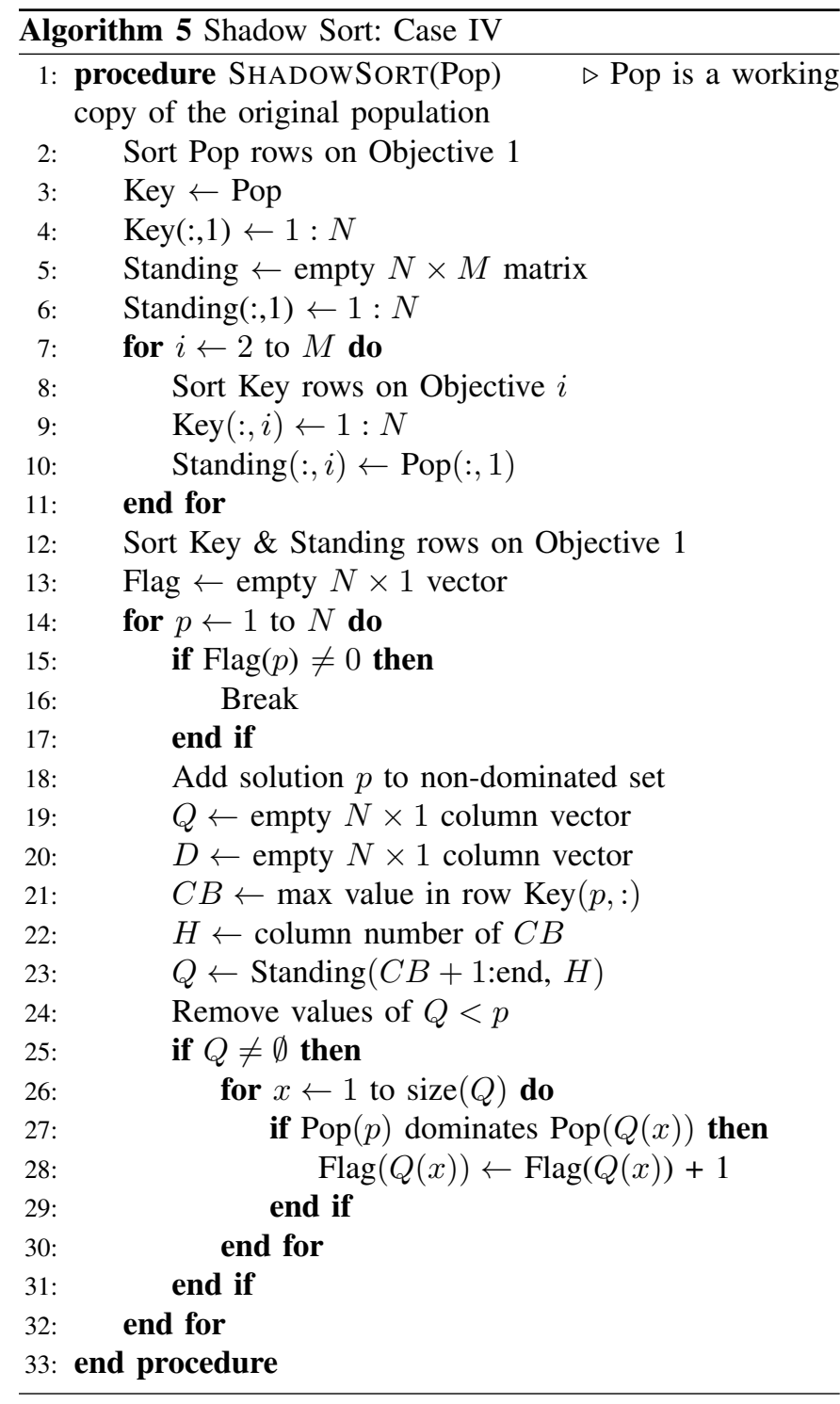

Sort (ENS) [1], Best Order Sort (BOS) [2] and Merge Nondominated Sort (MNDS) [3]. These three algorithms in turn have been tested and proven superior against other existing algorithms such as deductive sort (DS) [5] and corner sort (CS) [6]. The primary measure of algorithm performance is the time to sort. Three tests are conducted for comparison. In the first test, the algorithms perform non-dominated sorting on randomly generated large populations, which are created by initialising an $N \times M$ matrix with values between zero and one. These populations vary in size from 500 to 10000 in increments of 500 for 5, 10, 15 and 20 objectives (Fig. 4a to Fig. 4d). Two variations of this test were conducted, namely entire population sorting, as well as rank 1 sorting. Rank 1 sorting only identifies a population's rank 1 non-dominated solutions. This is a practical test because it relates to the case where many multi-objective optimization algorithms preserve only the best (or at most, a predefined number of) rank solutions to be used to generate the next generation of candidate solutions.

A second test is conducted where the population size is kept at 10000 , but the number of objectives is varied from two to
TABLE I

TEST 3: RESULTS OF SORTING THE NON-CONVEX POPULATION PRESENTED IN FIG. 6.

\begin{tabular}{|l|c|c|}
\cline { 2 - 3 } \multicolumn{1}{c|}{} & Mean (s) & SD (s) \\
\hline BOS & 24.84 & 5.47 \\
\hline ENS & 9.97 & 1.34 \\
\hline MNDS & 1.51 & 0.06 \\
\hline SS & 0.43 & 0.02 \\
\hline
\end{tabular}

20. As with the first test, this test is conducted for ranking of the entire population, as well as for rank 1 sorting. The results of Test 2 are shown in Fig. 5.

The results indicate that Shadow Sort is a faster nondominated sorting algorithm than BOS, ENS and MNDS on all population sizes and with any number of objectives (bar entire population sorting on 3-dimensional populations where ENS tests marginally faster). The difference in sorting time between entire population sorting and rank 1 sorting is large in populations with smaller dimensions. However, as the number of dimensions increases, the majority of a population's solutions exist as non-dominated solutions, and so it is understandable that there exists little difference in sorting time between entire population and rank 1 sorting.

An interesting phenomenon is seen in Test 2 for Shadow Sort, namely that the sorting time steadily increases until 17 objectives, after which the sorting time actually decreases. This is because it is at 17 objectives that Shadow Sort changes from Case III to Case IV where it is able to take advantage of the higher-dimensional setting as explained in Case IV.

As mentioned in Case II, Shadow Sort employs a special case for three-dimensional problems. Whilst this case is competitive against existing algorithms such as ENS (albeit it marginally slower), this case exhibits far superior performance in populations where a high percentage of the solutions are rank 1 solutions. An example population is shown graphically in Fig. 6.

Test 3 was conducted to evaluate the performance of Shadow Sort's Case II against such a population. As before, all four algorithms are tested against this population 30 times and the mean computation time reported. These results, along with their standard deviations (SD) are presented in Table I.

All three algorithms sorted the same 30 different populations, and the results were checked against each other to ensure that they were sorted identically. All tests were implemented using Matlab on an HP notebook with $1.8 \mathrm{GHz}$ Intel core i7 and 64 bit Windows 10 .

\section{CONCLUSION}

Presented was a novel, hybrid non-dominated sorting algorithm called Shadow Sort which uses one of four different sorting techniques depending on the number of objectives in the population. Each of the four cases were presented with their respective pseudo-code and illustrative small examples where appropriate. Shadow Sort was then compared against existing state-of-the-art algorithms ([1], [2] \& [3]) by means of three types of tests, each repeated 30 times. When searching only for rank 1 solutions, Shadow Sort proved to have the fastest sorting time for randomly generated populations for 


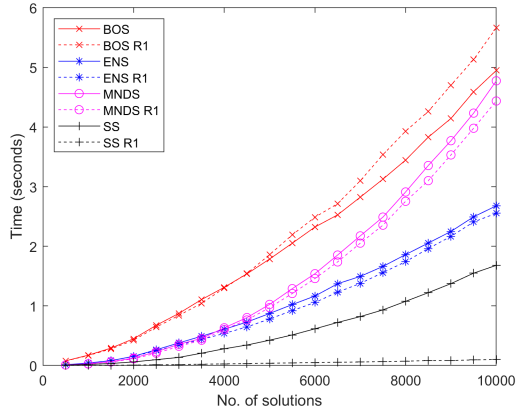

(a) Sorting times for populations with five objectives

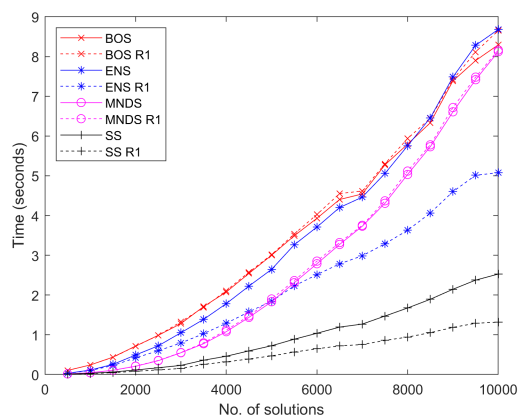

(b) Sorting times for populations with 10 objectives

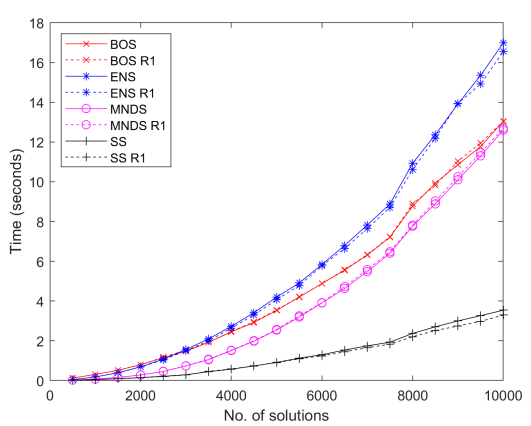

(c) Sorting times for populations with 15 objectives

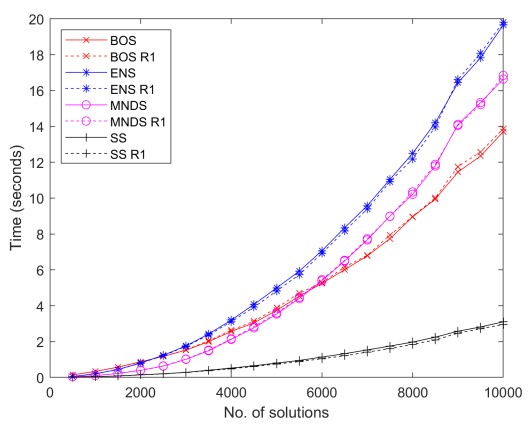

(d) Sorting times for populations with 20 objectives

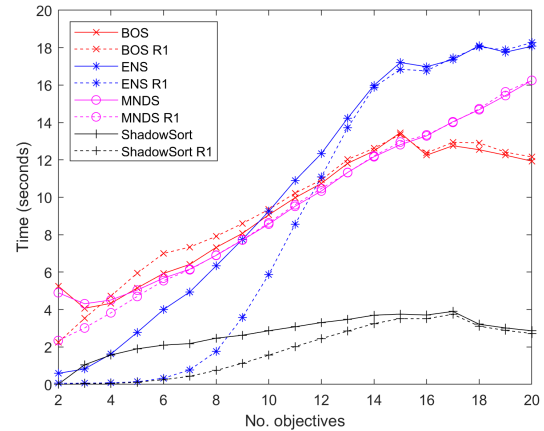

Fig. 5. Test 2: Sorting times for populations with 10000 solutions

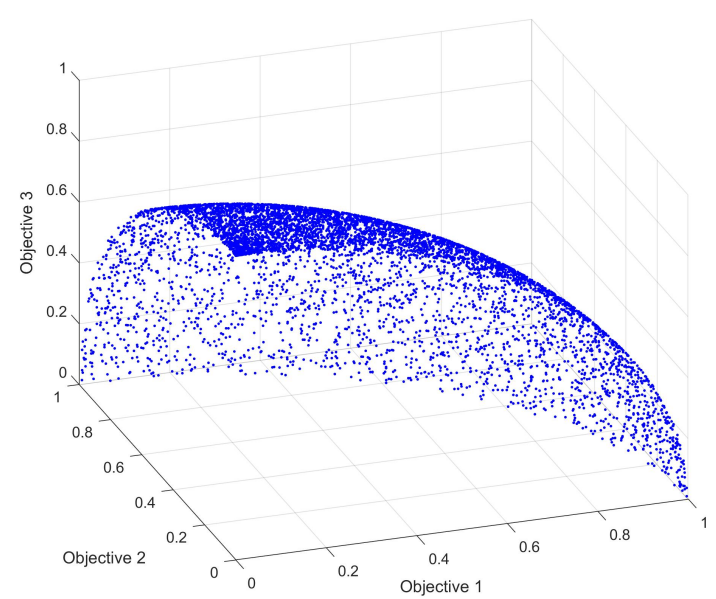

Fig. 6. A non-convex population where all solutions are non-dominated.

all population sizes (500 to 10000 in increments of 500), as well as for all problems with different numbers of objectives (2 to 20). This statement is also true when classifying all solutions in the population, with an exception to ENS [1] on three-dimensional randomly generated populations. The third test was employed to specifically evaluate the performance of Shadow Sort Case II, which achieves best results on threedimensional problems when a high percentage of the solutions form part of the rank 1 solutions.

In future work, Shadow Sort will be integrated with multiobjective optimization meta-heuristics. The quality of solutions sets will be evaluated to determine if better solutions can be obtained in shorter running times.

Fig. 4. Test 1 results for BOS, ENS, MNDS and SS. 


\section{REFERENCES}

[1] X. Zhang, Y. Tian, R. Cheng, and Y. Jin, "An efficient approach to nondominated sorting for evolutionary multiobjective optimization," IEEE Transactions on Evolutionary Computation, vol. 19, no. 2, pp. 201-213, 2014.

[2] P. C. Roy, M. M. Islam, and K. Deb, "Best order sort: a new algorithm to non-dominated sorting for evolutionary multi-objective optimization," in Proceedings of the 2016 on genetic and evolutionary computation conference companion, 2016, pp. 1113-1120.

[3] J. Moreno, D. Rodriguez, A. J. Nebro, and J. A. Lozano, "Merge nondominated sorting algorithm for many-objective optimization," IEEE Transactions on Cybernetics, 2020.

[4] S. Mishra, S. Saha, and S. Mondal, "Mbos: modified best order sort algorithm for performing non-dominated sorting," in 2018 IEEE Congress on Evolutionary Computation (CEC). IEEE, 2018, pp. 1-8.

[5] K. McClymont and E. Keedwell, "Deductive sort and climbing sort: New methods for non-dominated sorting," Evolutionary computation, vol. 20, no. 1, pp. 1-26, 2012.

[6] H. Wang and X. Yao, "Corner sort for pareto-based many-objective optimization," IEEE transactions on cybernetics, vol. 44, no. 1, pp. 92$102,2013$.

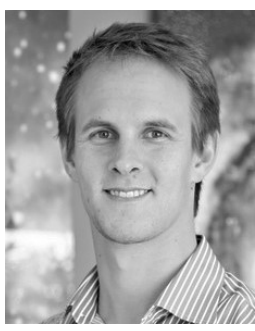

Nicholas Tränkle is an industrial engineer and a qualified commercial pilot. His research interests include algorithm improvement for multi-objective optimization problems.

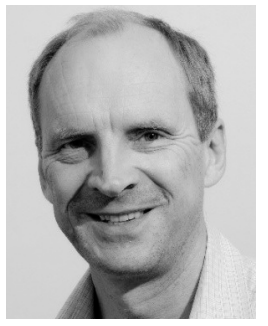

James Bekker is an associate professor in the department of industrial engineering, Stellenbosch University, South Africa. His research interests include multi-objective optimization of simulated stochastic problems. 